\title{
Subject Index Vol. 68, 2006
}

\section{ORL}

Accessory nerve function 88

Acoustic rhinometry 199

Active middle ear implant 378

Acute otitis media 135, 139

Adenoviral vector 220

Adhesive otitis media 195

Air and bone conduction 324

- conduction 319

Antidiuretic hormone 279

Artificial ear 115

Atresia 378

Auditory steady-state response 64

Auricle 378

Autologous fat 164

Basal cell carcinoma 83

Behavioral hearing assessment 64

Benign paroxysmal positioning vertigo 329

Body mass index 228

Bone conduction 319, 393

Calcium hydroxylapatite 103

- phosphate cement 103

Cautery-assisted uvulopalatoplasty 149

Cholesteatoma 237, 243, 296

Chromatography, high-pressure liquid 93

Cochlea 353, 359, 393

Cochlear amplifier 334,373

- - gain 347

- implant 77, 156, 270

- - benefits 283

- implantation 213

- mechanics 353, 359, 393

- model 365

Columella 324

Compensatory turbinate hypertrophy 199

Complications 259

Computer simulations 386

Concurrent chemoradiation therapy 206

Cordectomy 164

Corticosteroid dose 170

Cyclin-dependent kinase inhibitor 296

Cymetra 146

Cytokeratin 19177

- 20177

Dacryocystorhinostomy 266

Deep neck infection 259

Dexamethasone 185

- pharmacokinetics 93

Digital camera 302
Vol. 68, No. 1, 2006 has its own Subject Index

Distortion product otoacoustic emissions 334,340

Diuretics 170

Drug delivery 386

Ear malformation 378

Electromyography 88

Eosinophils 118

Finite element method 319

- elements 393

Force platform 232

Free otoliths 329

Frozen section 83

Gene delivery methods 220

Generalised circuit model 324

Glycerol test 279

Grommet insertion 115

Guinea pig labyrinth 93

Hair cells 353

Head and neck cancer 83, 189

Hearing 353, 359

- disorders, pediatrics 237

- loss 118, 378

Hemangiopericytoma 69

Hereditary hearing loss 57

5- $\mathrm{HT}_{2 \mathrm{~A}}$ receptor 123

Hydrodynamic cochlea model 347

Hypopharyngeal cancer 206

Implant fixation 270

Implantable hearing aid 378

Incus 243

Injectable material 103

Injection laryngoplasty 103

Inner ear 386

- - mechanics 329

Interferometry 353

Intracochlear pressure 359,365

Isosorbide 279

Juvenile nasopharyngeal angiofibroma 273

Lacrimal stenosis 266

Laryngeal carcinoma 177, 253

Laryngectomy 146

Laryngofissure 164

Laser-assisted uvulopalatoplasty 149

Lateral selective neck dissection 88
Leakage 146

Level IIB 88

Lipoma, parotid gland 290

Malleus 243

Mastoidectomy 156, 213

Mechanical response 365

Ménière's disease 279

MESNA 195

Microtia 378

Middle ear mechanics 324

- - surgery 243

Midfacial degloving 273

Minimal access surgery 270

- masking level 110

Mitochondrial hearing loss 57

Mitomycin C 73, 185

Modeling 302

Molecular detection 177

Mucosal wave 164

Multiple simultaneous stimuli 64

Multivariate logistic regression analysis 170

Myringotomy 73, 185

Nasal airflow 199

- endoscopy 129

Neck metastasis 177

Neocord tissue 164

Nonlinear cochlea models 340

Nonsyndromic hearing loss 57

Oral cavity tumor, murine orthotopic 159

Organ of Corti impedance 365

Osmotic diuretics 279

Ossicles 243

Ossicular chain 324

- reconstruction 243

Otitis media 195

- $\quad$ - with effusion 237

- - , intractable 118

- -, secretory 156

Otosclerosis 99

Outer hair cell(s) 365, 373

Outpatient surgery 99

Pain, postoperative 149

Parapharyngeal space 290

Parotid gland, lipoma 290

Partial laryngectomy 253

Penicillin resistant Streptococcus pneumoniae 139 
Penicillin-binding protein 139

Perilymph 93

Peripheral hearing organ 319

Pharmacokinetics 386

Plasma osmolality 279

Polymerase chain reaction 135, 139

Potassium channel 373

Powered instrumentation 129

Pre-operative embolization 273

Prestin 373

Quality of life 253

- $\quad$ - , elderly 283

Radiotherapy 253

Resorbable mesh reconstruction plate 77

Retraction pocket 195

Rhinoresistometry 199

Round window 378

- _ membrane 386

- _ - permeability 220

Selective neck dissection 177

Semicircular canals 329

Sensorineural hearing loss, acute low-tone 170
Septal deviation 199

Serial unstained celloidin sections 302

Serogroup 135

Serotonergic system 123

Serotype 135

Sinonasal hemangioma 69

Sleep apnea 123

Snoring 149

Sound therapy, mixing point 110

Speech-coding strategies 283

Sphenoid sinus 129

Sphenoidotomy 129

Squamos cell carcinoma 83

Squamous cell carcinoma 159

Stent 266

Steroids 386

Streptococcus pneumoniae 135

Sudden sensorineural hearing loss 247

Suprameatal approach 213

Surgical margins 83

- training 115

Syndromic hearing loss 57

Three-dimensional reconstruction 302

Time domain analysis 340

Tinnitus retraining therapy 110
Titanium mesh reconstruction plate 77

Tonsillar hypertrophy 228

Total laryngectomy 253

Tracheoesophageal puncture 146

Transtympanic steroid therapy 247

Traveling wave 347

Tumor volume 206

Tympanic membrane 185

- $\quad$, rat 73

Ultrasound, high-resolution 159

Unknown primary tumor 189

Uvulopalatopharyngoplasty 149

Vestibular end organs 302

- pathophysiology 329

Vestibulospinal reflex 232

Vocal fold medialization 103

Voice prosthesis 146

Wound healing 73, 185

X-linked hearing loss 57 